\title{
Remarks on Regularity Criteria for Axially Symmetric Weak Solutions to the Navier-Stokes Equations, II
}

\author{
Adam Kubica \\ November 17, 2018 \\ Faculty of Mathematics and Information Science \\ Warsaw University of Technology \\ ul. Koszykowa 75, Warsaw 00-662 \\ a.kubica@mini.pw.edu.pl
}

\begin{abstract}
We examine the conditional regularity of the solutions of Navier-Stokes equations in the entire three-dimensional space under the assumption that the data are axially symmetric. We show that if positive part of the radial component of velocity satisfies a weighted Serrin condition and in addition angular component satisfies some condition, then the solution is regular.
\end{abstract}

\section{Introduction}

Let us consider the Navier-Stokes equations in entire three-dimensional space

$$
\begin{array}{rcc}
\frac{\partial \mathbf{u}}{\partial t}+\mathbf{u} \cdot \nabla \mathbf{u}-\nu \Delta \mathbf{u}+\nabla p=\mathbf{0} & \text { in } & (0, T) \times \mathbb{R}^{3} \\
\operatorname{div} \mathbf{u}=0 & \text { in } & (0, T) \times \mathbb{R}^{3} \\
\mathbf{u}(0, \mathbf{x})=\mathbf{u}_{0}(\mathbf{x}) & \text { in } & \mathbb{R}^{3},
\end{array}
$$

where $\mathbf{u}:(0, T) \times \mathbb{R}^{3} \rightarrow \mathbb{R}^{3}$ is the velocity field, $p:(0, T) \times \mathbb{R}^{3} \rightarrow \mathbb{R}$ is the pressure, $0<T \leq \infty$, $\nu$ is the viscosity coefficient, $\mathbf{u}_{0}$ is the initial velocity and the forcing term is, for the sake of simplicity, considered to be zero. Our main result is following

Theorem 1. Let $\mathbf{u}$ be a weak solution to problem (1) satisfying the energy inequality with $\mathbf{u}_{0} \in W^{2,2}\left(\mathbb{R}^{3}\right)$ and $r u_{\theta}(0) \in L^{\infty}\left(\mathbb{R}^{3}\right)$. Let $\mathbf{u}_{0}$ be axisymmetric. If, in addition, $u_{r}^{+}$a positive part of radial component of velocity satisfies $r^{d} u_{r}^{+} \in L^{w, s}\left((0, T) \times\left(\mathbb{R}^{3} \cap\left\{r<\delta_{1}\right\}\right)\right)$ for some $s \in\left(\frac{3}{2}, \infty\right), w \in(1, \infty)$ and $d \in(-1,1)$ such that $\frac{2}{w}+\frac{3}{s}+d=1$ for some positive $\delta_{1}$ and $r^{1-\delta_{0}} u_{\theta} \in L^{\infty}\left((0, T) \times \mathbb{R}^{3}\right)$ for some positive $\delta_{0}$, then $(\mathbf{u}, p)$, where $p$ is the corresponding pressure, is an axisymmetric strong solution to problem (1) which is unique in the class of all weak solutions satisfying the energy inequality. 
It is convenient to write the equations (11) in cylindrical coordinates

$$
\begin{gathered}
u_{r, t}+u_{r} u_{r, r}+u_{z} u_{r, z}-\frac{1}{r} u_{\theta}^{2}+p_{, r}-\nu\left[\frac{1}{r}\left(r u_{r, r}\right)_{, r}+u_{r, z z}-\frac{u_{r}}{r^{2}}\right]=0, \\
u_{\theta, t}+u_{r} u_{\theta, r}+u_{z} u_{\theta, z}+\frac{1}{r} u_{\theta} u_{r}-\nu\left[\frac{1}{r}\left(r u_{\theta, r}\right)_{, r}+u_{\theta, z z}-\frac{u_{\theta}}{r^{2}}\right]=0, \\
u_{z, t}+u_{r} u_{z, r}+u_{z} u_{z, z}+p_{, z}-\nu\left[\frac{1}{r}\left(r u_{z, r}\right)_{, r}+u_{z, z z}\right]=0 .
\end{gathered}
$$

The equation of continuity has the following form in cylindrical coordinates

$$
u_{r, r}+\frac{u_{r}}{r}+u_{z, z}=0
$$

We put

$$
\boldsymbol{\omega}=\operatorname{curl} \mathbf{u}
$$

We have

$$
\omega_{r}=-u_{\theta, z}, \omega_{\theta}=u_{r, z}-u_{z, r}, \omega_{z}=u_{\theta, z}+\frac{u_{\theta}}{r}
$$

hence we get

$$
\begin{gathered}
\omega_{r, t}+u_{r} \omega_{r, r}+u_{z} \omega_{r, z}-u_{r, r} \omega_{r}-u_{r, z} \omega_{z}-\nu\left[\frac{1}{r}\left(r \omega_{r, r}\right)_{, r}+\omega_{r, z z}-\frac{\omega_{r}}{r^{2}}\right]=0, \\
\omega_{\theta, t}+u_{r} \omega_{\theta, r}+u_{z} \omega_{\theta, z}-\frac{u_{r}}{r} \omega_{\theta}+2 \frac{u_{\theta}}{r} \omega_{r}-\nu\left[\frac{1}{r}\left(r \omega_{\theta, r}\right)_{, r}+\omega_{\theta, z z}-\frac{\omega_{\theta}}{r^{2}}\right]=0, \\
\omega_{z, t}+u_{r} \omega_{z, r}+u_{z} \omega_{z, z}-u_{z, r} \omega_{r}-u_{z, z} \omega_{z}-\nu\left[\frac{1}{r}\left(r \omega_{z, r}\right)_{, r}+\omega_{z, z z}\right]=0 .
\end{gathered}
$$

Suppose that $0<t^{*}<T$ is the time of the first blow up of the solutions, i.e. the smaller positive number such that $\sup _{t \in\left(0, t^{*}\right)}\|\nabla \mathbf{u}(t, \cdot)\|_{L^{2}\left(\mathbb{R}^{3}\right)}=\infty$. Then, for $0<\bar{t}<t^{*}$ the equations (22)-(44) and (77)-(9) are satisfied in $\left((0, \bar{t}) \times \mathbb{R}^{3}\right)$ in strong sense. We will show that it is impossible, if $u_{r}^{+}$ and $u_{\theta}$ satisfy our assumptions.

First we multiply (3) by $\left|\frac{u_{\theta}}{r^{\mu}}\right|^{p-2} \frac{u_{\theta}}{r^{2 \mu}}$, then after integrating by parts we get

$\frac{1}{p} \frac{d}{d t} \|\left.\frac{u_{\theta}}{r^{\mu}}\right|_{p} ^{p}+\left.\left.\frac{4(p-1) \nu}{p^{2}} \int|\nabla| \frac{u_{\theta}}{r^{\mu}}\right|^{\frac{p}{2}}\right|^{2}+\nu\left(1-\mu^{2}\right) \int\left|\frac{u_{\theta}}{r^{\mu}}\right|^{p} \frac{1}{r^{2}}+(1+\mu) \int \frac{u_{r}^{+}}{r}\left|\frac{u_{\theta}}{r^{\mu}}\right|^{p}=(1+\mu) \int \frac{u_{r}^{-}}{r}\left|\frac{u_{\theta}}{r^{\mu}}\right|^{p}$.

Next, we multiply (8) by $\left|\frac{\omega_{\theta}}{r^{\alpha}}\right|^{q-2} \frac{\omega_{\theta}}{r^{2 \alpha}}$, then after integrating by parts we get 


$$
\begin{gathered}
\frac{1}{q} \frac{d}{d t}\left\|\frac{\omega_{\theta}}{r^{\alpha}}\right\|_{q}^{q}+\left.\left.\frac{4 \nu(q-1)}{q^{2}} \int|\nabla| \frac{\omega_{\theta}}{r^{\alpha}}\right|^{\frac{q}{2}}\right|^{2}+\nu\left(1-\alpha^{2}\right) \int\left|\frac{\omega_{\theta}}{r^{\alpha}}\right|^{q} \frac{1}{r^{2}}+(1-\alpha) \int \frac{u_{r}^{-}}{r}\left|\frac{\omega_{\theta}}{r^{\alpha}}\right|^{q} \\
=(1-\alpha) \int \frac{u_{r}^{+}}{r}\left|\frac{\omega_{\theta}}{r^{\alpha}}\right|^{q}+2 \int \frac{u_{\theta}}{r} u_{\theta, z}\left|\frac{\omega_{\theta}}{r^{\alpha}}\right|^{q-2} \frac{\omega_{\theta}}{r^{2 \alpha}} .
\end{gathered}
$$

Thus we have

$$
\begin{gathered}
\frac{1}{p} \frac{d}{d t}\left\|\frac{u_{\theta}}{r^{\mu}}\right\|_{p}^{p}+\frac{1}{q} \frac{d}{d t}\left\|\frac{\omega_{\theta}}{r^{\alpha}}\right\|_{q}^{q}+\left.\left.\frac{4(p-1) \nu}{p^{2}} \int|\nabla| \frac{u_{\theta}}{r^{\mu}}\right|^{\frac{p}{2}}\right|^{2}+\left.\left.\frac{4 \nu(q-1)}{q^{2}} \int|\nabla| \frac{\omega_{\theta}}{r^{\alpha}}\right|^{\frac{q}{2}}\right|^{2} \\
+\nu\left(1-\mu^{2}\right) \int\left|\frac{u_{\theta}}{r^{\mu}}\right|^{p} \frac{1}{r^{2}}+\nu\left(1-\alpha^{2}\right) \int\left|\frac{\omega_{\theta}}{r^{\alpha}}\right|^{q} \frac{1}{r^{2}}+(1+\mu) \int \frac{u_{r}^{+}}{r}\left|\frac{u_{\theta}}{r^{\mu}}\right|^{p}+(1-\alpha) \int \frac{u_{r}^{-}}{r}\left|\frac{\omega_{\theta}}{r^{\alpha}}\right|^{q} \\
=(1+\mu) \int \frac{u_{r}^{-}}{r}\left|\frac{u_{\theta}}{r^{\mu}}\right|^{p}+(1-\alpha) \int \frac{u_{r}^{+}}{r}\left|\frac{\omega_{\theta}}{r^{\alpha}}\right|^{q}+2 \int \frac{u_{\theta}}{r} u_{\theta, z}\left|\frac{\omega_{\theta}}{r^{\alpha}}\right|^{q-2} \frac{\omega_{\theta}}{r^{2 \alpha}} \equiv(1+\mu) I_{1}+(1-\alpha) I_{2}+2 I_{3} .
\end{gathered}
$$

\section{Estimate of $I_{3}$}

Proposition 1. For $\gamma \in(0,3), \quad q \in\left(\frac{2}{4-\gamma}, 2\right), \quad p=\frac{(4-\gamma) q}{2}, \quad \mu \in(-1,1)$ and $a \in(0,1)$ we have

$$
\left|I_{3}\right| \leq \varepsilon_{1} \int\left|\frac{u_{\theta}}{r^{\mu}}\right|^{p-2}\left|\frac{u_{\theta, z}}{r^{\mu}}\right|^{2}+\varepsilon_{2} \int\left|\frac{u_{\theta}}{r^{\mu}}\right|^{p} \frac{1}{r^{2}}+\varepsilon_{3} \int\left|\frac{\omega_{\theta}}{r^{\alpha}}\right|^{q} \frac{1}{r^{2}}+C \int\left|\frac{\omega_{\theta}}{r^{\alpha}}\right|^{q},
$$

where

$$
\alpha=2 \mu-\frac{\gamma}{2}(1+\mu)-\frac{2(q-1)}{q}(1-a),
$$

and $C=C\left(\gamma, q, a, \varepsilon_{1}, \varepsilon_{2}, \varepsilon_{3}\right)$.

Proof. We have

$$
\begin{gathered}
I_{3}=\int \frac{u_{\theta}}{r} u_{\theta, z}\left|\frac{\omega_{\theta}}{r^{\alpha}}\right|^{q-2} \frac{\omega_{\theta}}{r^{2 \alpha}} \leq \int\left|\frac{u_{\theta}}{r^{\mu}}\right|^{\frac{p}{2}-1}\left|\frac{u_{\theta, z}}{r^{\mu}}\right| \cdot \frac{\left|u_{\theta}\right|^{2-\frac{p}{2}}}{r^{1+\alpha-\frac{\mu p}{2}}}\left|\frac{\omega_{\theta}}{r^{\alpha}}\right|^{q-1} \\
\stackrel{Y(2,2)}{\leq} \varepsilon_{1} \int\left|\frac{u_{\theta}}{r^{\mu}}\right|^{p-2}\left|\frac{u_{\theta, z}}{r^{\mu}}\right|^{2}+C\left(1 / \varepsilon_{1}\right) \int \frac{\left|u_{\theta}\right|^{4-p}}{r^{2\left[1+\alpha-\frac{\mu p}{2}\right]}\left|\frac{\omega_{\theta}}{r^{\alpha}}\right|^{2(q-1)}} \\
=\varepsilon_{1} \int\left|\frac{u_{\theta}}{r^{\mu}}\right|^{p-2}\left|\frac{u_{\theta, z}}{r^{\mu}}\right|^{2}+C\left(1 / \varepsilon_{1}\right) \int\left|r u_{\theta}\right|^{\gamma} \cdot \frac{\left|u_{\theta}\right|^{4-p-\gamma}}{r^{2+2 \alpha-\mu p+\gamma-\frac{4(q-1)}{q} a}} \cdot \frac{\left|\omega_{\theta}\right|^{2(q-1) a}}{r^{2 \alpha(q-1) a+\frac{4(q-1)}{q} a}} \cdot \frac{\left|\omega_{\theta}\right|^{2(q-1)(1-a)}}{r^{2 \alpha(q-1)(1-a)}} .
\end{gathered}
$$

Applying Young inequality with exponents $\left(\infty, \frac{q}{2-q}, \frac{q}{2(q-1) a}, \frac{q}{2(q-1)(1-a)}\right)$ we get 


$$
\left|I_{3}\right| \leq \varepsilon_{1} \int\left|\frac{u_{\theta}}{r^{\mu}}\right|^{p-2}\left|\frac{u_{\theta, z}}{r^{\mu}}\right|^{2}+\varepsilon_{2} \int \frac{\left|u_{\theta}\right|^{[4-p-\gamma] \frac{q}{2-q}}}{r^{\frac{q}{2-q}\left[2+2 \alpha-\mu p+\gamma-\frac{4(q-1)}{q} a\right]}}+\varepsilon_{3} \int\left|\frac{\omega_{\theta}}{r^{\alpha}}\right|^{q} \frac{1}{r^{2}}+C \int\left|\frac{\omega_{\theta}}{r^{\alpha}}\right|^{q},
$$

where $C$ depends on $\varepsilon_{1}, \varepsilon_{2}, \varepsilon_{3}, a$ and $\left\|r u_{\theta}\right\|_{L^{\infty}}$. But we have $[4-p-\gamma] \frac{q}{2-q}=p$ and $\frac{q}{2-q}\left[2+2 \alpha-\mu p+\gamma-\frac{4(q-1)}{q} a\right]=p \mu+2$.

\section{Estimate of $I_{1}$}

Remark 1. For all $q \in(1, \infty), \quad \alpha$ and $\varepsilon_{0}$ satisfy $-2+\varepsilon_{0}<\alpha<\varepsilon_{0}$. Then there exists a constant $C=C\left(q, \alpha, \varepsilon_{0}\right)$ such that

$$
\int\left|\frac{u_{r}}{r^{1+\alpha}}\right|^{q} \cdot \frac{1}{r^{2-\varepsilon_{0} q}} \leq C \int\left|\frac{\omega_{\theta}}{r^{\alpha}}\right|^{q} \cdot \frac{1}{r^{2-\varepsilon_{0} q}}
$$

Proof. We have $\left\|\frac{u_{r}}{r}\right\|_{q} \leq c(q)\left\|\omega_{\theta}\right\|_{q}$ for all $q \in(1, \infty)$. So we have to verify that $r^{-q\left(\alpha+\frac{2}{q}-\varepsilon_{0}\right)}$ is $A_{q}$ weight. This holds if

$$
-2<-q\left(\alpha+\frac{2}{q}-\varepsilon_{0}\right)<2(q-1)
$$

i.e. $-2+\varepsilon_{0}<\alpha<\varepsilon_{0}$.

Proposition 2. Assume that $\varpi \equiv\left\|r^{1-\delta_{0}} u_{\theta}\right\|_{L^{\infty}} \leq C$ for some $\delta_{0} \in\left(0, \frac{1}{3}\right)$. Then for all $\gamma \in(0,3), q \in\left(\frac{2}{4-\gamma}, 2\right), a \in\left(1-\frac{(4-\gamma) q^{2}}{4(q-1) \delta_{0}}, 1\right) \cap(0,1)$,

$$
\mu \in\left(q \delta_{0}-1, q \delta_{0}+\frac{\gamma}{4-\gamma}\right) \cap(-1,1)
$$

and for $\varepsilon_{4}, \varepsilon_{5} \in(0,1)$ the following estimate holds

$$
\left|I_{1}\right|=\int \frac{u_{r}^{-}}{r}\left|\frac{u_{\theta}}{r^{\mu}}\right|^{p} \leq \varepsilon_{4} \int\left|\frac{u_{\theta}}{r^{\mu}}\right|^{p} \frac{1}{r^{2}}+\varepsilon_{5} \int\left|\frac{\omega_{\theta}}{r^{\alpha}}\right|^{q} \frac{1}{r^{2}}+C \int\left|\frac{\omega_{\theta}}{r^{\alpha}}\right|^{q},
$$

where $p=\frac{(4-\gamma) q}{2}, \quad \alpha=2 \mu-\frac{\gamma}{2}(1+\mu)-\frac{2(q-1)}{q}(1-a), \quad$ and $C=C\left(\varepsilon_{4}, \varepsilon_{5}, a, q, \delta_{0}, \gamma, \varpi\right)$.

Proof. We denote $\kappa=-\frac{2(q-1)}{q}(1-a)$. Then we can write

$$
\begin{gathered}
I_{3}=\int \frac{u_{r}^{-}}{r}\left|\frac{u_{\theta}}{r^{\mu}}\right|^{p}=\int\left|\frac{u_{\theta}}{r^{\mu+\frac{2}{p}}}\right|^{\frac{p(q-1)}{q}} \cdot\left|r^{1-\delta_{0}} u_{\theta}\right|^{\frac{p}{q}} \cdot \frac{u_{r}^{-}}{r^{1+\alpha+\frac{2}{q}-\kappa-\frac{p}{q} \delta_{0}}} \stackrel{Y\left(\frac{q}{q-1}, q\right)}{\leq} \\
\leq \varepsilon_{4} \int\left|\frac{u_{\theta}}{r^{\mu}}\right|^{p} \frac{1}{r^{2}}+C\left(q, \gamma, \varpi, \varepsilon_{4}\right) \int\left|\frac{u_{r}}{r^{1+\alpha}}\right|^{q} \frac{1}{r^{2-\kappa q-\delta_{0} p}} .
\end{gathered}
$$

We define $\varepsilon_{0}$ by equality $-\kappa q-\delta_{0} p=-q\left[\frac{(4-\gamma) q}{2} \delta_{0}-\frac{2(q-1)}{q}(1-a)\right] \equiv-q \varepsilon_{0}$. Then using (16) we deduce that $-2+\varepsilon_{0}<\alpha<\varepsilon_{0}$, hence we can use remark 1 and we get 


$$
I_{3} \leq \varepsilon_{4} \int\left|\frac{u_{\theta}}{r^{\mu}}\right|^{p} \frac{1}{r^{2}}+C\left(q, \gamma, \varpi, \varepsilon_{4}, a, \mu\right) \int\left|\frac{\omega_{\theta}}{r^{\alpha}}\right|^{q} \frac{1}{r^{2-\varepsilon_{0} q}}
$$

Using the assumption on $a$ we get that $b=1-\frac{q \varepsilon_{0}}{2}$ satisfies $b \in(0,1)$ and we can write

$$
\int\left|\frac{\omega_{\theta}}{r^{\alpha}}\right|^{q} \frac{1}{r^{2-\varepsilon q}}=\int\left|\frac{\omega_{\theta}}{r^{\alpha+\frac{2}{q}}}\right|^{b q} \cdot\left|\frac{\omega_{\theta}}{r^{\alpha}}\right|^{(1-b) q} \stackrel{Y\left(\frac{1}{b}, \frac{1}{1-b}\right)}{\leq} \varepsilon_{5} \int\left|\frac{\omega_{\theta}}{r^{\alpha}}\right|^{q} \frac{1}{r^{2}}+C\left(1 / \varepsilon_{5}\right) \int\left|\frac{\omega_{\theta}}{r^{\alpha}}\right|^{q} .
$$

Thus we get

$$
\left|I_{3}\right| \leq \varepsilon_{4} \int\left|\frac{u_{\theta}}{r^{\mu}}\right|^{p} \frac{1}{r^{2}}+\varepsilon_{5} \int\left|\frac{\omega_{\theta}}{r^{\alpha}}\right|^{q} \frac{1}{r^{2}}+C \int\left|\frac{\omega_{\theta}}{r^{\alpha}}\right|^{q},
$$

where $C=C\left(q, \gamma, \varpi, \varepsilon_{4}, \varepsilon_{5}, a, \mu\right)$.

From propositions 1 and 2 we get

Corollary 1. Assume that $\varpi \equiv\left\|r^{1-\delta_{0}} u_{\theta}\right\|_{L^{\infty}} \leq C$ for some $\delta_{0} \in\left(0, \frac{1}{3}\right)$. Then for all $\gamma \in(0,3)$, $q \in\left(\frac{2}{4-\gamma}, 2\right), a \in\left(1-\frac{(4-\gamma) q^{2}}{4(q-1)} \delta_{0}, 1\right)$,

$$
\mu \in\left(q \delta_{0}-1, q \delta_{0}+\frac{\gamma}{4-\gamma}\right) \cap(-1,1)
$$

and for $\varepsilon_{1}, \varepsilon_{2}, \varepsilon_{3} \in(0,1)$ the following estimate holds

$$
\left|I_{1}\right|+\left|I_{3}\right| \leq \varepsilon_{1} \int\left|\frac{u_{\theta}}{r^{\mu}}\right|^{p} \frac{1}{r^{2}}+\varepsilon_{2} \int\left|\frac{\omega_{\theta}}{r^{\alpha}}\right|^{p} \frac{1}{r^{2}}+\left.\left.\varepsilon_{3} \int|\nabla| \frac{u_{\theta}}{r^{\mu}}\right|^{\frac{p}{2}}\right|^{2}+C \int\left|\frac{\omega_{\theta}}{r^{\alpha}}\right|^{p},
$$

where $p=\frac{(4-\gamma) q}{2}, \quad \alpha=2 \mu-\frac{\gamma}{2}(1+\mu)-\frac{2(q-1)}{q}(1-a), \quad$ and $C=C\left(\varepsilon_{1}, \varepsilon_{2}, \varepsilon_{3}, a, q, \delta_{0}, \gamma, \varpi\right)$.

Corollary 2. Assume that $\varpi \equiv\left\|r^{1-\delta_{0}} u_{\theta}\right\|_{L^{\infty}}<\infty$ for some $\delta_{0} \in\left(0, \frac{1}{3}\right)$. Then for $\varepsilon \in\left(0, \frac{1}{14}\right)$ such that

$$
\left(\frac{1-2 \varepsilon}{1-\varepsilon}\right) \varepsilon \leq \delta_{0}
$$

for all $\varepsilon_{1}, \varepsilon_{2}, \varepsilon_{3} \in(0,1)$ the following estimate holds

$$
\left|I_{1}\right|+\left|I_{3}\right| \leq \varepsilon_{1} \int\left|\frac{u_{\theta}}{r^{\mu}}\right|^{p} \frac{1}{r^{2}}+\varepsilon_{2} \int\left|\frac{\omega_{\theta}}{r^{\alpha}}\right|^{p} \frac{1}{r^{2}}+\left.\left.\varepsilon_{3} \int|\nabla| \frac{u_{\theta}}{r^{\mu}}\right|^{\frac{p}{2}}\right|^{2}+C \int\left|\frac{\omega_{\theta}}{r^{\alpha}}\right|^{p},
$$

where $p=2\left(1-\varepsilon^{2}\right), q=2(1-\varepsilon), \mu=\frac{1-\varepsilon}{1+\varepsilon}$ and $\alpha=-2(1-2 \varepsilon)(1+\varepsilon) \varepsilon$ and $C=C\left(\varepsilon_{1}, \varepsilon_{2}, \varepsilon_{3}, \delta_{0}, \varpi, \varepsilon\right)$. In particular, for such exponents we have

$$
\int\left|\frac{u_{r}}{r^{1+\alpha}}\right|^{q} \leq c(q, \alpha) \int\left|\frac{\omega_{\theta}}{r^{\alpha}}\right|^{q}
$$

and

$$
\frac{2}{\infty}+\frac{3}{q}-1-\alpha \leq \frac{1}{2}+7 \varepsilon<1
$$


Proof. We have to verify the assumptions of corollary 1. Therefore we put $\gamma=2(1-\varepsilon)$. Then $\gamma \in(0,3)$ and $q \in\left(\frac{2}{4-\gamma}, 2\right)$ and we set $a=1-2\left(1-\varepsilon^{2}\right) \varepsilon$. Then using (20) we get $a \in\left(1-\frac{(4-\gamma) q^{2}}{4(q-1)} \delta_{0}, 1\right)$. Finally, $\mu=\frac{1-\varepsilon}{1+\varepsilon}$ satisfies (18), because $\delta_{0}$ is positive. Then we get (19) with $p=\frac{(4-\gamma) q}{2}=2\left(1-\varepsilon^{2}\right)$ and $\alpha=2 \mu-\frac{\gamma}{2}(1+\mu)-\frac{2(q-1)}{q}(1-a)=-2(1-2 \varepsilon)(1+\varepsilon) \varepsilon$.

In order to get (22) we have to verify that $r^{-q \alpha}$ is $\mathcal{A}_{q}$ weight. Indeed, in our case we have $\frac{2}{q}-2<\alpha<\frac{2}{q}$. Inequality (23) we obtain by direct calculations.

\section{Estimate of $I_{2}$}

Proposition 3. Assume that for some positive $\delta_{1}$ holds $t \mapsto f(t) \equiv\left[\int_{\mathbb{R}^{3} \cap\left\{r<\delta_{1}\right\}}\left|r^{d} u_{r}^{+}\right|^{s} d x\right]^{\frac{w}{s}}$ is integrable for some $s \in\left(\frac{3}{2}, \infty\right), w \in(1, \infty)$ and $d \in(-1,1)$ such that $\frac{2}{w}+\frac{3}{s}+d=1$. Then for $q \in(1, \infty)$ and $\alpha \in(-1,1)$ and for all $\varepsilon_{1}, \varepsilon_{2} \in(0,1)$ the following estimate holds

$$
\left|I_{2}\right| \leq \varepsilon_{1} \int\left|\frac{\omega_{\theta}}{r^{\alpha}}\right|^{q} \frac{1}{r^{2}}+\left.\left.\varepsilon_{2} \int|\nabla| \frac{\omega_{\theta}}{r^{\alpha}}\right|^{\frac{q}{2}}\right|^{2}+C[f(t)+g(t)] \int\left|\frac{\omega_{\theta}}{r^{\alpha}}\right|^{q},
$$

where $C=C\left(\varepsilon_{1}, \varepsilon_{2}, \delta_{1}, s, w, q\right)$ and $g(t)=\int\left|u_{r}^{+}\right|^{\frac{10}{3}}$.

Weak solutions belong to $L^{\frac{10}{3}}$, thus function $g(t)$ is integrable.

Proof. Let $\eta=\eta(r)$ be smooth cut off function such that $\eta(r)=1$ for $r<\delta_{1} / 2$ and $\eta(r)=0$ for $r>\delta_{1}$. The we have

$$
I_{2}=\int \frac{\eta u_{r}^{+}}{r}\left|\frac{\omega_{\theta}}{r^{\alpha}}\right|^{q}+\int \frac{(1-\eta) u_{r}^{+}}{r}\left|\frac{\omega_{\theta}}{r^{\alpha}}\right|^{q} \equiv I_{2,0}+I_{2,1} .
$$

We begin with the first integral. We denote $a=\frac{2}{2-\left(\frac{2}{w}+\frac{3}{s}\right)}, \quad b=\frac{2 s}{w}+3$. Then $a>1$ and $b>3$ and we can write

$$
\begin{gathered}
I_{2,0} \equiv \int \frac{\eta u_{r}^{+}}{r}\left|\frac{\omega_{\theta}}{r^{\alpha}}\right|^{q}=\int\left|\frac{\omega_{\theta}}{r^{\alpha}}\right|^{\frac{q}{a}} \frac{1}{r^{\frac{2}{a}}} \cdot \eta u_{r}^{+} r^{\frac{2-a}{a}}\left|\frac{\omega_{\theta}}{r^{\alpha}}\right|^{q \frac{a-1}{a}} \\
\stackrel{Y\left(a, \frac{a}{a-1}\right)}{\leq} \varepsilon_{1} \int\left|\frac{\omega_{\theta}}{r^{\alpha}}\right|^{q} \frac{1}{r^{2}}+c\left(\varepsilon_{1}, a\right) \int\left|\eta u_{r}^{+}\right|^{\frac{a}{a-1}} r^{\frac{2-a}{a-1}} \cdot\left|\frac{\omega_{\theta}}{r^{\alpha}}\right|^{q} .
\end{gathered}
$$

Now we estimate the last integral on the right hand side

$$
\begin{aligned}
& \int\left|\eta u_{r}^{+}\right|^{\frac{a}{a-1}} r^{\frac{2-a}{a-1}} \cdot\left|\frac{\omega_{\theta}}{r^{\alpha}}\right|^{q H\left(\frac{b}{2}, \frac{b}{b-2}\right)} \leq\left[\int\left|\eta u_{r}^{+}\right|^{\frac{a b}{2(a-1)}} r^{\frac{b(2-a)}{2(a-1)}}\right]^{\frac{2}{b}} \cdot\left[\int\left|\frac{\omega_{\theta}}{r^{\alpha}}\right|^{\frac{q b}{b-2}}\right]^{\frac{b-2}{b}} \\
& =\left[\int\left|\eta u_{r}^{+}\right|^{\frac{a b}{2(a-1)}} r^{\frac{b(2-a)}{2(a-1)}}\right]^{\frac{2}{b}} \cdot\left[\int\left|\frac{\omega_{\theta}}{r^{\alpha}}\right|^{q \frac{b-3}{b-2}} \cdot\left|\frac{\omega_{\theta}}{r^{\alpha}}\right|^{\frac{3 q}{b-2}}\right]^{\frac{b-2}{b}} \\
& \stackrel{H\left(\frac{b-2}{b-3}, b-2\right)}{\leq}\left[\int\left|\eta u_{r}^{+}\right|^{\frac{a b}{2(a-1)}} r^{\frac{b(2-a)}{2(a-1)}}\right]^{\frac{2}{b}} \cdot\left[\int\left|\frac{\omega_{\theta}}{r^{\alpha}}\right|^{q}\right]^{\frac{b-3}{b}} \cdot\left[\int\left|\frac{\omega_{\theta}}{r^{\alpha}}\right|^{3 q}\right]^{\frac{1}{b}}
\end{aligned}
$$




$$
\stackrel{Y\left(\frac{b}{3}, \frac{b}{b-3}\right)}{\leq} \varepsilon_{2}\left[\int\left|\frac{\omega_{\theta}}{r^{\alpha}}\right|^{3 q}\right]^{\frac{1}{3}}+c\left(\varepsilon_{2}, b\right)\left[\int\left|\eta u_{r}^{+}\right|^{\frac{a b}{2(a-1)}} r^{\frac{b(2-a)}{2(a-1)}}\right]^{\frac{2}{b-3}} \cdot\left[\int\left|\frac{\omega_{\theta}}{r^{\alpha}}\right|^{q}\right] .
$$

By definition we have $\frac{a b}{2(a-1)}=s, \frac{b(2-a)}{2(a-1)}=d s$ and $\frac{2}{b-3}=\frac{w}{s}$, thus

$$
I_{2,0} \leq \varepsilon_{1} \int\left|\frac{\omega_{\theta}}{r^{\alpha}}\right|^{q} \frac{1}{r^{2}}+\varepsilon_{2}\left[\int\left|\frac{\omega_{\theta}}{r^{\alpha}}\right|^{3 q}\right]^{\frac{1}{3}}+c\left(\varepsilon_{1}, \varepsilon_{2}, w, s\right) f(t) \cdot\left[\int\left|\frac{\omega_{\theta}}{r^{\alpha}}\right|^{q}\right] .
$$

In order to estimate $I_{2,1}$ we put $a=4$ and $b=5$ and then proceeding analogously we get

$$
I_{2,1} \leq \varepsilon_{1} \int\left|\frac{\omega_{\theta}}{r^{\alpha}}\right|^{q} \frac{1}{r^{2}}+\varepsilon_{2}\left[\int\left|\frac{\omega_{\theta}}{r^{\alpha}}\right|^{3 q}\right]^{\frac{1}{3}}+c\left(\varepsilon_{1}, \varepsilon_{2}\right)\left[\int\left|(1-\eta) u_{r}^{+}\right|^{\frac{10}{3}} r^{-\frac{5}{3}}\right] \cdot\left[\int\left|\frac{\omega_{\theta}}{r^{\alpha}}\right|^{q}\right] .
$$

Clearly $\int\left|(1-\eta) u_{r}^{+}\right|^{\frac{10}{3}} r^{-\frac{5}{3}} \leq\left(2 / \delta_{1}\right)^{5 / 3} \int\left|u_{r}^{+}\right|^{\frac{10}{3}}$ and the last function in integrable1 1 on $(0, T)$. Finally, applying Sobolev imbedding theorem in estimates (25) and (26) we get (24).

Corollary 3. Assume that $\varpi \equiv\left\|r^{1-\delta_{0}} u_{\theta}\right\|_{L^{\infty}} \leq C$ for some $\delta_{0} \in\left(0, \frac{1}{3}\right)$ and

$$
t \mapsto f(t) \equiv\left[\int_{\mathbb{R}^{3} \cap\left\{r<\delta_{1}\right\}}\left|r^{d} u_{r}^{+}\right|^{s} d x\right]^{\frac{w}{s}} \text { is integrable on }(0, T)
$$

for some $s \in\left(\frac{3}{2}, \infty\right), w \in(1, \infty)$ and $d \in(-1,1)$ such that $\frac{2}{w}+\frac{3}{s}+d=1$ and $\delta_{1}$ positive. Then for $\varepsilon \in\left(0, \frac{1}{14}\right)$ such that

$$
\left(\frac{1-2 \varepsilon}{1-\varepsilon}\right) \varepsilon \leq \delta_{0}
$$

the following estimate holds

$$
\begin{aligned}
& \frac{d}{d t}\left\|\frac{u_{\theta}}{r^{\mu}}\right\|_{p}^{p}+\frac{d}{d t}\left\|\frac{\omega_{\theta}}{r^{\alpha}}\right\|_{q}^{q}+\left.\left.\frac{4(p-1) \nu}{p} \int|\nabla| \frac{u_{\theta}}{r^{\mu}}\right|^{\frac{p}{2}}\right|^{2}+\left.\left.\frac{4 \nu(q-1)}{q} \int|\nabla| \frac{\omega_{\theta}}{r^{\alpha}}\right|^{\frac{q}{2}}\right|^{2} \\
& +\nu\left(1-\mu^{2}\right) \int\left|\frac{u_{\theta}}{r^{\mu}}\right|^{p} \frac{1}{r^{2}}+\nu\left(1-\alpha^{2}\right) \int\left|\frac{\omega_{\theta}}{r^{\alpha}}\right|^{q} \frac{1}{r^{2}} \leq C[1+f(t)+g(t)]\left\|\frac{\omega_{\theta}}{r^{\alpha}}\right\|_{q}^{q},
\end{aligned}
$$

where $p=2\left(1-\varepsilon^{2}\right), q=2(1-\varepsilon), \mu=\frac{1-\varepsilon}{1+\varepsilon}$ and $\alpha=-2(1-2 \varepsilon)(1+\varepsilon) \varepsilon$ and $C=C\left(\nu, \varepsilon, \delta_{0}, \delta_{1}, \varpi, s, w\right)$ and $g(t)=\int\left|u_{r}^{+}\right|^{\frac{10}{3}}$. In particular,

$$
\underset{t \in(0, T)}{\operatorname{ess} \sup _{0}}\left\|\frac{\omega_{\theta}}{r^{\alpha}}\right\|_{q} \leq C^{\prime}\left[\left\|\frac{u_{\theta}}{r^{\mu}}(0)\right\|_{p}+\left\|\frac{\omega_{\theta}}{r^{\alpha}}(0)\right\|_{q}\right]
$$

where $C^{\prime}=C^{\prime}\left(C,\|f\|_{L^{1}(0, T)}\right)$ and $\mathbf{u}$ is regular on $(0, T)$.

Proof. Under our assumption we can use corollary 2 and proposition 3 and we get (29) and by Gronwall lemma we obtain (30). Then using inequality (22) we get $\underset{t \in(0, T)}{\operatorname{ess} \sup }\left\|\frac{u_{r}}{r^{1+\alpha}}\right\|_{q} \leq$ const. and (23) holds. Therefore we can apply theorem 1 [2] and we deduce the regularity of $\mathbf{u}$.

\footnotetext{
${ }^{1}$ Weak solutions belong to $L^{\frac{10}{3}}$.
} 
Remark 2. The condition (27) can be weakened a bit. Namely it is enough to assume that

$$
t \mapsto \widetilde{f}(t) \equiv \frac{\left[\int\left|r^{d} u_{r}^{+}\right|^{s} d x\right]^{\frac{w}{s}}}{1+\ln ^{+}\left(\left\|\frac{u_{\theta}}{r^{\mu}}\right\|_{p}^{p}+\left\|\frac{\omega_{\theta}}{r^{\alpha}}\right\|_{q}^{q}\right)} \quad \text { is integrable on }(0, T),
$$

where $d, w, s, p, q$ are as above. Indeed, from (29) we have

$$
\frac{d}{d t}\left(\left\|\frac{u_{\theta}}{r^{\mu}}\right\|_{p}^{p}+\left\|\frac{\omega_{\theta}}{r^{\alpha}}\right\|_{q}^{q}\right) \leq C[1+f(t)]\left(\left\|\frac{u_{\theta}}{r^{\mu}}\right\|_{p}^{p}+\left\|\frac{\omega_{\theta}}{r^{\alpha}}\right\|_{q}^{q}\right)
$$

so arguing similarly as in [4] we can write

$$
\frac{d}{d t} \ln \left[1+\ln ^{+}\left(\left\|\frac{u_{\theta}}{r^{\mu}}\right\|_{p}^{p}+\left\|\frac{\omega_{\theta}}{r^{\alpha}}\right\|_{q}^{q}\right)\right] \leq C \frac{[1+f(t)]}{1+\ln ^{+}\left(\left\|\frac{u_{\theta}}{r^{\mu}}\right\|_{p}^{p}+\left\|\frac{\omega_{\theta}}{r^{\alpha}}\right\|_{q}^{q}\right)} .
$$

After integrating with respect time we obtain the bound for $\operatorname{ess~}_{t \in(0, T)}\left\|\frac{\omega_{\theta}}{r^{\alpha}}\right\|_{q}$.

\section{References}

[1] O. Kreml, M. Pokorný: A regularity criterion for the angular velocity component in axisymmetric Navier-Stokes equations, Electron. J. Differential Equations 2007, No. 08, 10 pp.

[2] A. Kubica, M. Pokorný, W. Zajączkowski, Remarks on regularity criteria for axially symmetric weak solutions to the Navier-Stokes equations, Math. Methods Appl. Sci. 35 (2012), no. 3, 360-371.

[3] S. Leonardi, J. Málek, J. Nečas, M. Pokorný: On axially symmetric flows in $\mathbb{R}^{3}$, ZAA 18 (1999), 639-649.

[4] S. Montgomery-Smith, Conditions implying regularity of the three dimensional NavierStokes equation, Appl. Math. 50 (2005), no. 5, 451-464.

[5] J. Neustupa, A. Novotný, P. Penel: An interior regularity of a weak solution to the Navier-Stokes equations in dependence on one component of velocity, Topics in mathematical fluid mechanics Quad. Mat. 10 (2002), 163-183.

[6] J. Neustupa, M. Pokorný, An interior regularity criterion for an axially symmetric suitable weak solution to the Navier-Stokes equations, J. Math. Fluid Mech. 2 (2000), no. 4, 381-399.

[7] J. Neustupa, M. Pokorný: Axisymmetric flow of Navier-Stokes fluid in the whole space with non-zero angular velocity component, Math. Boh. 126, No. 2 (2001), 469-481.

[8] P. Penel, M. Pokorný: Some new regularity criteria for the Navier-Stokes equations containing the gradient of velocity, Appl. Math. 49 (2004), 483-493. 
[9] M. Pokorný, A regularity criterion for the angular velocity component in the case of axisymmetric Navier-Stokes equations, Elliptic and parabolic problems (Rolduc/Gaeta, 2001), 233-242, World Sci. Publ., River Edge, NJ, 2002.

[10] M. Pokorný: On the result of He concerning the smoothness of solutions to the Navier-Stokes equations, Electron. J. Diff. Eqns 11 (2003), 1-8.

[11] M. Pokorný: A short note on regularity criteria for the Navier-Stokes equations containing the velocity gradient, Regularity and other Aspects of the Navier-Stokes Equations vol 70 (Warsaw: Banach Center Publications) pp 199-207, 2005.

[12] M.R. Uchovskii, B.I. Yudovich: Axially symmetric flows of an ideal and viscous fluid in the whole space (in Russian, also J. Appl. Math. Mech, 32 (1968), 52-61), Prikladnaya matematika i mechanika 32 (1968), 59-69. 